\title{
Studies of Some Lanthanide(III) Nitrate Complexes of Schiff Base Ligands
}

\author{
KISHOR ARORA* MUKESH SHARMA and K.P. SHARMA ${ }^{\S}$ \\ *Dept. of Chemistry, Govt. K.R.G. Auto. P.G. College, Gwalior (M.P.), India. \\ Dept. of Chemistry, G.L.S. College, Banmore, Morena (M.P.), India. \\ ${ }^{\S}$ Dept. of Chemistry, S.M.S. Govt. Science College, Gwalior (M.P.), India. \\ kishorarora@rediffmail.com
}

Received 17 April 2009; Accepted 10 June 2009

\begin{abstract}
The studies of 16 new lanthanide(III) nitrate complexes of Schiff base ligands are discussed. Schiff bases were obtained by the condensation of 2-methyl-4-N,N-bis-2'-cyanoethyl aminobenzaldehyde with aniline and 3 different substituted anilines. Lanthanide(III) nitrates, viz. gadolinium(III) nitrate, lanthanum(III) nitrate, samarium(III) nitrate and cerium(III) nitrate were chosen to synthesize new complexes. The complexes were characterized on the basis of physicochemical studies viz. elemental analysis, spectral, viz. IR and electronic spectral and magnetic studies. TGA studies of some of the representative complexes were also done. Some of the representative complexes were also screened for the anti microbial studies.
\end{abstract}

Keywords: Lanthanide(III) Nitrate complexes, Schiff bases, Physicochemical studies.

\section{Introduction}

Lanthanides or lanthanons form a longest series of the periodic table. It is $4 f$-inner transition series. Lanthanide(III) ions, because of their size and charge are the best ions to form stable complexes with high coordination number ${ }^{1-5}$. Coordination compounds of lanthanide in which lanthanide ions exhibit coordination number 6 to 10 are reported $^{6-7}$.

Schiff base metal complexes have played a major role in the development of coordination chemistry ${ }^{8-11}$. In this work, we wish to report lanthanide(III) nitrate complexes with some Schiff base ligands shown in the Figure 1. 


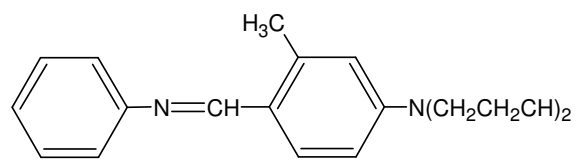

[2- Methyl-4-NN-bis-2'-cyanoethylaminobenzylidine aniline]

(MCEABAB) (I)<smiles>CCCN(CC)c1ccc(C=Nc2ccc(Cl)cc2)c(C)c1</smiles>

[2- Methyl-4-NN-bis-2'-cyanoethylaminobenzylidine $p$-chloro aniline]

(MCEABCAB) (II)<smiles>CCN(CC)c1ccc(C=Nc2ccc(C)cc2)c(C)c1</smiles>

[2- Methyl-4-NN-bis-2'-cyanoethylaminobenzylidine $p$-toludine]

(MCEABPT) (III)

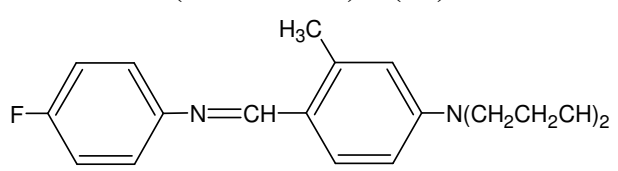

[2- Methyl-4-NN-bis-2'- cyanoethylaminobenzylidine $p$-fluoroaniline]

(MCEABFAB) (IV)

\section{Experimental}

Figure 1. Structure of Schiff base ligands I to IV.

Lanthanide(III) nitrates viz. lanthanum(III) nitrate, cerium(III) nitrate, samarium(III) nitrate and gadolinium(III) nitrate were obtained from B.D.H./C.D.H. and used as such. Solvents were obtained from various sources such as B.D.H., E-Merck and S.D. Fine chemicals and were used as such or after distillation if felt necessary.

\section{Preparation of ligands}

Schiff base ligands were prepared by either of the following methods ${ }^{12-13}$.

\section{$\operatorname{Method}(A)$}

A solution of the aldehyde $(1 \mathrm{mmol})$ in absolute alcohol $(50 \mathrm{~mL})$ was mixed with corresponding amine i.e. (i) aniline (ii) $p$-fluoroaniline $(1: 1 \mathrm{mmol})$ in the same solvent and two drops of piperidine were added. The mixture was refluxed for $4 \frac{1}{2} \mathrm{~h}$. On cooling dark coloured solids separated, which were filtered under suction and recrystallised from ethanol as pale/ yellow solids.

\section{$\operatorname{Method}(B)$}

A mixture of the aldehyde $(1 \mathrm{mmol})$ and corresponding amine i.e. (i) $p$-chloroaniline (ii) $p$-toludine $(1: 1 \mathrm{mmol})$ was taken in a round bottom flask to which two drops of piperidine were added. 
The contents were heated for $4 \frac{1}{2} \mathrm{~h}$ in an oil bath maintained at $105-110{ }^{0} \mathrm{C}$. The contents first melted and then solidified. The Schiff bases were purified by recrystallisation from ethanol as pale/yellow solids. Physical characteristics of the Schiff base ligands are listed in Table 1.

Table 1. Physical characteristics of the ligands.

\begin{tabular}{clcccc}
\hline S. No. & Name of ligands & Yield, $\%$ & State & Colour & M.P. ${ }^{0}$ C \\
\hline 1 & MCEABAB & $85 \%$ & Solid & Light yellow & $140-142$ \\
2 & MCEABCAB & $80 \%$ & Solid & Light yellow & $142-144$ \\
3 & MCEABPT & $82 \%$ & Solid & Pale Yellow & $143-146$ \\
4 & MCEABFAB & $87.5 \%$ & Solid & Pale Yellow & $138-140$ \\
\hline
\end{tabular}

\section{Synthesis of complexes}

Complexes were prepared by treating corresponding lanthanide(III) nitrate $(1 \mathrm{mmol})$ in isopropanol with ligand solution $(1: 4 \mathrm{mmol})$ in the same solvent. In some cases complexes were isolated immediately in cold while in other cases in hot solution. In some cases resulting solution were refluxed on the water bath for $1-3 \mathrm{~h}\left(\mathrm{Ca} 70-75{ }^{\circ} \mathrm{C}\right)$. The resulting complexes were collected after filtration washed with isopropanol and finally with ether and dried in vacuo over $\mathrm{CaCl}_{2}{ }^{14}$.

\section{Results and Discussion}

Interaction of lanthanide(III) nitrates viz. lanthanum(III) nitrate, cerium(III) nitrate, samerium(III) nitrate and gadolinium nitrate(III) with Schiff base ligands results in the formation of complexes with general composition $\mathrm{Ln}(\mathrm{L})_{4}\left(\mathrm{NO}_{3}\right)_{3}$ [Where $\mathrm{Ln}=\mathrm{La}, \mathrm{Ce}, \mathrm{Sm}$ and $\mathrm{Gd}$ and $\mathrm{L}=\mathrm{MCEABAB}, \mathrm{MCEABCAB}, \mathrm{MCEABPT}$ and MCEABFAB] The general equation for the preparation of the complexes is shown below:

$$
\mathrm{M}\left(\mathrm{NO}_{3}\right)_{3}+4 \mathrm{~L} \underset{\text { Heat, reflux }}{\stackrel{\text { Isopropanol }}{\longrightarrow}} \mathrm{M}(\mathrm{L})_{4}\left(\mathrm{NO}_{3}\right)_{3}
$$

\section{[M = La, Ce, Sm \& Gd, L= MCEABAB, MCEABCAB, MCEABPT and MCEABFAB]}

The elemental analysis of these complexes are given in Table 2. New complexes are fairly stable and can be stored for a long period. All are sufficiently soluble in common organic solvents and are non hygroscopic in nature. These complexes show almost negligible electrical conductivity in $\mathrm{PhNO}_{2}$ medium which shows their non electrolytic nature ${ }^{15}$.

The molecular weight data of the complexes also support this fact. Magnetic moment data show that lanthanum(III) nitrate complexes are essentially diamagnetic in nature while all other complexes are paramagnetic due to presence of $4 f$-electrons which are effectively shielded by $5 s^{2} p^{2}$ electrons ${ }^{16}$. This shows that $4 f$-electrons do not participate in the bond formation ${ }^{17}$. Magnetic moment data of the complexes are assembled in Table 2.

\section{Spectroscopic studies}

\section{Infrared Spectral studies}

The I.R. Spectra were recorded in the $4000-400 \mathrm{~cm}^{-1}$ range using $\mathrm{KBr}$ pallets. IR spectra of the Schiff base ligands were analysed by comparison of the spectra of substituted benzene and the corresponding assignments were listed with comparing the IR spectra of previously reported data ${ }^{18}$. 
Table 2. Analytical, conductance, molecular weight and magnetic moment data for lanthanide(III) nitrate complexes of Schiff base ligands under Study.

\begin{tabular}{|c|c|c|c|c|c|c|c|c|c|c|}
\hline \multirow{2}{*}{ S. No. } & \multirow{2}{*}{ Complexes } & \multirow{2}{*}{ Colour } & \multirow{2}{*}{$\begin{array}{c}\text { Yield } \\
\%\end{array}$} & \multirow{2}{*}{$\underset{{ }^{0} \mathrm{C}}{\mathrm{M} . \mathrm{P}}$} & \multicolumn{3}{|c|}{ Analytical found (Calcd.)\% } & \multirow{2}{*}{$\begin{array}{c}{ }^{\wedge} \mathrm{M}, \\
\mathrm{ohm}^{-1} \\
\mathrm{~cm}^{2} \\
\mathrm{~mol}^{-1} \\
\end{array}$} & \multirow{2}{*}{$\begin{array}{l}\text { Mol. Wt. } \\
\text { calcd. } \\
\text { (Found) }\end{array}$} & \multirow{2}{*}{$\begin{array}{c}\text { Magnetic } \\
\text { Moment } \\
\text { (B.M.) }\end{array}$} \\
\hline & & & & & $\mathrm{C}$ & $\mathrm{H}$ & $\mathrm{N}$ & & & \\
\hline 1 & $\mathrm{La}(\mathrm{MCEABAB})_{4}\left(\mathrm{NO}_{3}\right)_{3}$ & Green & 79 & 152 & $56.71(60.45)$ & $5.32(5.03)$ & $15.27(16.75)$ & 3.1 & $1588(1598)$ & Diamag. \\
\hline 2 & $\mathrm{Ce}(\mathrm{MCEABAB})_{4}\left(\mathrm{NO}_{3}\right)_{3}$ & Yellow & 73 & 154 & $58.34(60.37)$ & $5.25(5.03)$ & $15.62(16.72)$ & 3.3 & $1590(1596)$ & 2.72 \\
\hline 3 & $\mathrm{Sm}(\mathrm{MCEABAB})_{4}\left(\mathrm{NO}_{3}\right)_{3}$ & Green & 80 & 152 & $62.37(60.00)$ & $5.69(5.00)$ & $15.96(16.62)$ & 3.2 & $1600(1610)$ & 1.54 \\
\hline 4 & $\mathrm{Gd}(\mathrm{MCEABAB})_{4}\left(\mathrm{NO}_{3}\right)_{3}$ & Yellow & 74 & 155 & $56.13(59.73)$ & $4.98(4.97)$ & $14.11(16.55)$ & 3.4 & $1607(1620)$ & 7.66 \\
\hline 5 & $\mathrm{La}(\mathrm{MCEABCAB})_{4}\left(\mathrm{NO}_{3}\right)_{3}$ & Light Brown & 68 & 150 & $54.7(55.42)$ & $4.85(4.38)$ & $14.90(15.35)$ & 4.0 & $1732(1742)$ & Diamag. \\
\hline 6 & $\mathrm{Ce}(\mathrm{MCEABCAB})_{4}\left(\mathrm{NO}_{3}\right)_{3}$ & Pale Yellow & 78 & 152 & $56.40(55.36)$ & $4.72(4.38)$ & $14.95(15.34)$ & 4.1 & $1734(1744)$ & 2.81 \\
\hline 7 & $\mathrm{Sm}(\mathrm{MCEABCAB})_{4}\left(\mathrm{NO}_{3}\right)_{3}$ & Light Yellow & 69 & 150 & $58.45(56.04)$ & $4.75(4.35)$ & $14.54(15.25)$ & 4.3 & $1744(1752)$ & 1.48 \\
\hline 8 & $\mathrm{Gd}(\mathrm{MCEABCAB})_{4}\left(\mathrm{NO}_{3}\right)_{3}$ & Brown & 79 & 158 & $56.42(54.82)$ & $6.07(4.34)$ & $14.02(15.19)$ & 4.4 & $1751(1760)$ & 7.79 \\
\hline 9 & $\mathrm{La}(\mathrm{MCEABPT})_{4}\left(\mathrm{NO}_{3}\right)_{3}$ & Dark Brown & 73 & 157 & $59.89(61.23)$ & $5.64(5.34)$ & $15.44(16.16)$ & 4.3 & $1646(1650)$ & Diamag. \\
\hline 10 & $\mathrm{Ce}(\mathrm{MCEABPT})_{4}\left(\mathrm{NO}_{3}\right)_{3}$ & Orange & 80 & 152 & $60.65(61.16)$ & $6.09(5.33)$ & $15.95(16.14)$ & 3.6 & $1648(1656)$ & 2.81 \\
\hline 11 & $\mathrm{Sm}(\mathrm{MCEABPT})_{4}\left(\mathrm{NO}_{3}\right)_{3}$ & Blackish & 75 & 160 & $59.30(60.83)$ & $5.23(5.31)$ & $15.90(16.05)$ & 3.9 & $1657(1665)$ & 1.62 \\
\hline 12 & $\mathrm{Gd}(\mathrm{MCEABPT})_{4}\left(\mathrm{NO}_{3}\right)_{3}$ & Dark Green & 74 & 150 & $58.90(60.54)$ & $5.20(5.28)$ & $15.89(15.97)$ & 4.1 & $1665(1675)$ & 7.81 \\
\hline 13 & $\mathrm{La}(\mathrm{MCEABFAB})_{4}\left(\mathrm{NO}_{3}\right)_{3}$ & Green & 70 & 155 & $59.94(57.76)$ & $5.21(4.57)$ & $15.36(16.00)$ & 4.3 & $1662(1670)$ & Diamag. \\
\hline 14 & $\mathrm{Ce}(\mathrm{MCEABFAB})_{4}\left(\mathrm{NO}_{3}\right)_{3}$ & Pale Yellow & 74 & 154 & $57.59(57.72)$ & $5.03(4.57)$ & $15.12(15.99)$ & 4.2 & $1663(1672)$ & 2.76 \\
\hline 15 & $\mathrm{Sm}(\mathrm{MCEABFAB})_{4}\left(\mathrm{NO}_{3}\right)_{3}$ & Light Green & 78 & 158 & $56.43(57.38)$ & $5.11(4.54)$ & $15.02(15.89)$ & 3.6 & $1673(1680)$ & 1.73 \\
\hline 16 & $\mathrm{Gd}(\mathrm{MCEABFAB})_{4}\left(\mathrm{NO}_{3}\right)_{3}$ & Dark Green & 81 & 152 & $59.29(57.18)$ & $5.24(4.52)$ & $14.88(15.82)$ & 3.4 & $1681(1688)$ & 7.91 \\
\hline
\end{tabular}


In case of complexes a notable peak $1599-1584 \mathrm{~cm}^{-1}$ of ligands shows negative shift and appears in the range of $\left[1530-1505 \mathrm{~cm}^{-1}\right]$ This notable peak is attributed to azomenthine $v(C=N)$ [19-20] stretching and its shift indicates that in complexes coordination of ligands in complexes is through azo methinic nitrogen atom ${ }^{21-22}$. The bands assigned to benzene ring vibration compared well with those of mono-substituted derivatives of benzene ${ }^{23}$ several other modes associated with C-H out of plane deformation appear in the region [764-754 cm $\mathrm{cm}^{-1}$. Some new medium to weak bands were also observed in the range (496 to $470 \mathrm{~cm}^{-1}$ ) in case of complexes ligands have no absorption. These new medium to weak bands are assigned as $y(\mathrm{Ln}-\mathrm{N})$ modes observed for various lanthanide(III) nitrate complexes. The partial IR data for various ligands and their corresponding complexes are given in Table 3.

Table 3. Partial IR spectral data for lanthanide(III) nitrate complexes of Schiff base ligands

\begin{tabular}{|c|c|c|c|}
\hline S. No. & Complexes & $\begin{array}{c}v(\mathrm{C}=\mathrm{N}) \\
\text { Stretching[Azomethine] }\end{array}$ & $\begin{array}{c}v(\mathrm{M}-\mathrm{N}) \text { (Metal- } \\
\text { Ligand Vibration) }\end{array}$ \\
\hline 1 & MCEABAB & $1584 \mathrm{~s}$ & - \\
\hline 2 & $\mathrm{La}(\mathrm{MCEABAB})_{4}\left(\mathrm{NO}_{3}\right)_{3}$ & $1511 \mathrm{~m}$ & $470 \mathrm{~m}$ \\
\hline 3 & $\mathrm{Ce}(\mathrm{MCEABAB})_{4}\left(\mathrm{NO}_{3}\right)_{3}$ & $1510 \mathrm{sh}$ & $475 \mathrm{~m}$ \\
\hline 4 & $\mathrm{Sm}(\mathrm{MCEABAB})_{4}\left(\mathrm{NO}_{3}\right)_{3}$ & $1530 \mathrm{w}$ & $480 \mathrm{~m}$ \\
\hline 5 & $\mathrm{Gd}(\mathrm{MCEABAB})_{4}\left(\mathrm{NO}_{3}\right)_{3}$ & $1525 \mathrm{w}$ & $485 \mathrm{w}$ \\
\hline 6 & MCEABCAB & $1599 \mathrm{~s}$ & - \\
\hline 7 & $\mathrm{La}(\mathrm{MCEABCAB})_{4}\left(\mathrm{NO}_{3}\right)_{3}$ & $1510 \mathrm{~m}$ & $472 \mathrm{w}$ \\
\hline 8 & $\mathrm{Ce}(\mathrm{MCEABCAB})_{4}\left(\mathrm{NO}_{3}\right)_{3}$ & $1530 \mathrm{w}$ & $476 \mathrm{~m}$ \\
\hline 9 & $\mathrm{Ce}(\mathrm{MCEABCAB})_{4}\left(\mathrm{NO}_{3}\right)_{3}$ & $1530 \mathrm{w}$ & $485 \mathrm{w}$ \\
\hline 10 & $\mathrm{Gd}(\mathrm{MCEABCAB})_{4}\left(\mathrm{NO}_{3}\right)_{3}$ & $1512 \mathrm{~W}$ & $473 \mathrm{w}$ \\
\hline 11 & MCEABPT & $1591 \mathrm{~s}$ & - \\
\hline 12 & $\mathrm{La}(\mathrm{MCEABPT})_{4}\left(\mathrm{NO}_{3}\right)_{3}$ & $1512 \mathrm{w}$ & $486 \mathrm{w}$ \\
\hline 13 & $\mathrm{Ce}(\mathrm{MCEABPT})_{4}\left(\mathrm{NO}_{3}\right)_{3}$ & $1509 \mathrm{~m}$ & $470 \mathrm{~m}$ \\
\hline 14 & $\mathrm{Sm}(\mathrm{MCEABPT})_{4}\left(\mathrm{NO}_{3}\right)_{3}$ & $1512 \mathrm{~s}$ & $481 \mathrm{~m}$ \\
\hline 15 & $\mathrm{Gd}(\mathrm{MCEABPT})_{4}\left(\mathrm{NO}_{3}\right)_{3}$ & $1512 \mathrm{~m}$ & $490 \mathrm{w}$ \\
\hline 16 & MCEABFAB & $1590 \mathrm{~s}$ & - \\
\hline 17 & $\mathrm{La}(\mathrm{MCEABFAB})_{4}\left(\mathrm{NO}_{3}\right)_{3}$ & $1511 \mathrm{~m}$ & $490 \mathrm{w}$ \\
\hline 18 & $\mathrm{Ce}(\mathrm{MCEABFAB})_{4}\left(\mathrm{NO}_{3}\right)_{3}$ & $1520 \mathrm{w}$ & $495 \mathrm{w}$ \\
\hline 19 & $\mathrm{Sm}(\mathrm{MCEABFAB})_{4}\left(\mathrm{NO}_{3}\right)_{3}$ & $1521 \mathrm{sh}$ & $490 \mathrm{~m}$ \\
\hline 20 & $\mathrm{Gd}(\mathrm{MCEABFAB})_{4}\left(\mathrm{NO}_{3}\right)_{3}$ & $1505 \mathrm{~m}$ & $496 \mathrm{~m}$ \\
\hline
\end{tabular}

It has been found ${ }^{24}$ that the separation of $\left(v_{2}+v_{5}\right)$ and $\left(v_{2}+v_{3}\right)$ is greater in bi-dentate (about 30-40 $\mathrm{cm}^{-1}$ ) than in monodentate (about $10 \mathrm{~cm}^{-1}$ ) nitrate complexes. The separation of $\left(v_{2}+v_{1}\right)$ and $\left(v_{2}+v 7_{4}\right)$ frequencies is greater for bi-dentate nitrate anions (about 200-300 cm$~^{-1}$ ) as compared to mono-dentate nitrate anions (about 10-200 $\mathrm{cm}^{-1}$ ) complex containing ionic nitrate groups show only one band in this region assignable to $\left(v_{1}+v_{3}\right)$. The occurrence of two absorptions at $1525-1480 \mathrm{~cm}^{-1}$ and $1325-1283 \mathrm{~cm}^{-1}$ region is attributed to $v_{4}$ and $v_{1}$ modes which suggest that nitrate anions in the complexes under study are covalently bonded and are present inside the coordination sphere ${ }^{25}$ If $\left(v_{4}-v_{1}\right)$ difference is taken as an approximate measure of covalence of nitrate groups a value of $\left[\sim 200 \mathrm{~cm}^{-1}\right]$ for the complexes under study suggest a strong covalency nature of nitrate in the complexes. Lever separation rule ${ }^{26}$ states that $b i$-dentate coordination of nitrate anion involves a greater distortion from $\mathrm{D}_{3} \mathrm{~h}$ symmetry than uni-dentate coordination. In case of spectra of complexes, under study it has been found that the separation of $\left(v_{1}+v_{4}\right)$ is good enough and is in the order $\left[\sim 200 \mathrm{~cm}^{-1}\right]$ this 
shows that according to Lever separation rule, nitrate ion may be considered as covalently bonded to metal ions in bi-dentate fashion. Infrared absorption frequencies $\left(\mathrm{cm}^{-1}\right)$ of nitrato groups of lanthanide(III) nitrate complexes of Schiff base are listed in Table 4.

Table 4. Infrared absorption frequencies $\left(\mathrm{cm}^{-1}\right)$ of nitrato groups of lanthanide(III) nitrate complexes

\begin{tabular}{cccccc}
\hline$v_{4}$ & $v_{1}$ & $v_{2}$ & $v_{6}$ & $v_{3}$ & $v_{5}$ \\
\hline $1525-$ & $1325-$ & $1022-$ & $835-$ & $764-$ & $705-$ \\
$1480 \mathrm{~cm}^{-1}$ & $1283 \mathrm{~cm}^{-1}$ & $1012 \mathrm{~cm}^{-1}$ & $810 \mathrm{~cm}^{-1}$ & $754 \mathrm{~cm}^{-1}$ & $665 \mathrm{~cm}^{-1}$ \\
\hline
\end{tabular}

\section{Electronic spectral studies}

Electronic spectral studies of lanthanide(III) metal complexes are significant and are important tool for the measurement of covalency in complexes. The line like spectra of lanthanide(III) metal compound appearing in the UV-visible and near IR regions arise from electronic transitions within the $4 f$-levels which are normal forbidden(27-28) but may become allowed after removal of degeneracy of $4 \mathrm{f}$-orbitals by external crystal field ${ }^{29-30}$.

Lanthanum(III) complexes has no significant absorption in visible region. The absorption bands of samarium(III) in visible and near IR region may appear due to transitions from ground levels ${ }^{4} \mathrm{H}_{5 / 2}$ to the excited J. levels.

The shift of hypersensitive bands has been utilized to calculate the nephelauxetic effect $(\beta)$, Sinha's covalency parameter $(\delta \%)$ (metal ligand covalency percentage) and the covalency factor $\left(b^{1} / 2\right)$ along with covalency angular overlap parameter $(\eta)$ these parameters have been calculated using the following expressions ${ }^{31}$.

$$
\begin{aligned}
\mathrm{b}^{1 / 2} & =1 / 2\left[(1-\beta)^{1 / 2}\right] \\
\delta \% & =[(1-\beta) / \beta] \times 100 \\
\eta & =(1-\beta 1 / 2) \beta 1 / 2
\end{aligned}
$$

The electronic spectral studies of lanthanide(III) metal complexes yield positive value for $(1-\beta)$ and $(\delta \%)$ which suggest that the bonding between metal and ligand is covalent in the complexes. The values of parameter of bonding $(\beta 1 / 2)$ and angular overlap parameter $(\eta)$ are also found to be positive indicating covalent bonding complexes. The electronic spectral data are presented in Table 6 .

\section{Thermogravimetric analysis}

Thermal studies of two representative complexes have been done successfully and their thermal decomposition patterns in different stages have also being studied. The decomposition data for the complexes is presented in Table 6 and 7. The complexes decompose by loosing ligand in parts in different heating stags and ultimately in the final step metal oxide are found in both the cases at high temperature ${ }^{32-33}$.

\section{Anti microbial studies}

Antimicrobial viz. antifungal and antibacterial studies of some of the representative complexes were carried out successfully. Results of these studies are included in Table 8 and 9. The studies were carried out on, Aspergillus fumigatus, Aspergillus niger and Mucor species among fungi and E.coli species in bacteria using paper disc method on appropriate nutrient medium. The results are included in Table 8 and 9. The colony diameters were measured after $48 \mathrm{~h}$ of incubation [La(MCEABAB $\left.)_{4}\left(\mathrm{NO}_{3}\right)_{3}\right]$ and [Ce(MCEABAB $)_{4}\left(\mathrm{NO}_{3}\right)_{3}$ ] were found to be effective against E.coli and [Sm(MCEABFAB $)_{4}\left(\mathrm{NO}_{3}\right)_{3}$ ] were found to be active against fungal species ${ }^{14}$. 
Table 5. Electronic spectral data $\left(\mathrm{cm}^{-1}\right)$ and related bonding parameter of lanthanide(III) nitrate complexes of Schiff bases.

\begin{tabular}{|c|c|c|c|c|c|c|c|c|c|c|}
\hline $\begin{array}{l}\text { S. } \\
\text { No. }\end{array}$ & & plexes & $\begin{array}{l}\text { Lanthanide } \\
\text { Salts, } \mathrm{cm}^{-1}\end{array}$ & $\begin{array}{l}\text { Complex } \\
\text { band } \mathrm{cm}^{-1}\end{array}$ & Energy Levels & $(1-b)$ & $\mathrm{b}$ & $b^{1 / 2}$ & $\mathrm{~d} \%$ & $\mathrm{~h}$ \\
\hline \multirow[b]{2}{*}{1} & \multirow{2}{*}{\multicolumn{2}{|c|}{$\mathrm{Sm}(\mathrm{MCEABAB})_{4}\left(\mathrm{NO}_{3}\right)_{3}$}} & 24850 & 24390 & ${ }^{4} \mathrm{H}_{5 / 2} \rightarrow{ }^{4} \mathrm{~F}_{9 / 2}$ & 0.018511 & 0.981489 & 0.136055 & 1.88601 & 0.009212 \\
\hline & & & 24100 & 23809 & $\rightarrow{ }^{6} \mathrm{P}_{5 / 2}$ & 0.012074 & 0.98792 & 0.109881 & 1.22216 & 0.0060216 \\
\hline \multirow{4}{*}{2} & \multirow{4}{*}{\multicolumn{2}{|c|}{$\mathrm{Sm}(\mathrm{MCEABCAB})_{4}\left(\mathrm{NO}_{3}\right)_{3}$}} & 21600 & 20833 & $\rightarrow{ }^{4} \mathrm{I}_{13 / 2}$ & 0.035509 & 0.96449 & 0.188438 & 3.68163 & 0.0175945 \\
\hline & & & 24850 & 24154 & ${ }^{4} \mathrm{H}_{5 / 2} \rightarrow{ }^{4} \mathrm{~F}_{9 / 2}$ & 0.028008 & 0.97199 & 0.1673552 & 2.88151 & 0.0139055 \\
\hline & & & 24100 & 23809 & $\rightarrow{ }^{6} \mathrm{P}_{5 / 2}$ & 0.012074 & 0.98792 & 0.109881 & 1.22216 & 0.0060216 \\
\hline & & & 21600 & 21097 & $\rightarrow{ }^{4} \mathrm{I}_{13 / 2}$ & 0.023287 & 0.97671 & 0.152600 & 2.38422 & 0.0115764 \\
\hline \multirow{2}{*}{3} & \multirow{2}{*}{\multicolumn{2}{|c|}{$\mathrm{Sm}(\mathrm{MCEABPT})_{4}\left(\mathrm{NO}_{3}\right)_{3}$}} & 24850 & 24271 & ${ }^{4} \mathrm{H}_{5 / 2} \rightarrow{ }^{4} \mathrm{~F}_{9 / 2}$ & 0.023299 & 0.97670 & 0.15264 & 2.38548 & 0.0115813 \\
\hline & & & 24100 & 23584 & $\rightarrow{ }^{6} \mathrm{P}_{5 / 2}$ & 0.02141 & 0.97859 & 0.146321 & 2.18784 & 0.0106471 \\
\hline \multirow{4}{*}{4} & \multirow{4}{*}{\multicolumn{2}{|c|}{$\mathrm{Sm}(\mathrm{MCEABFAB})_{4}\left(\mathrm{NO}_{3}\right)_{3}$}} & 21600 & 21367 & $\rightarrow{ }^{4} \mathrm{I}_{13 / 2}$ & 0.010787 & 0.98921 & 0.103860 & 1.09046 & 0.0053804 \\
\hline & & & 24850 & 23923 & ${ }^{4} \mathrm{~F}_{9 / 2} \longrightarrow{ }^{4} \mathrm{~F}_{9 / 2}$ & 0.037303 & 0.962697 & 0.193139 & 3.87484 & 0.0184742 \\
\hline & & & 24100 & 23809 & $\rightarrow{ }^{6} \mathrm{P}_{5 / 2}$ & 0.012075 & 0.98792 & 0.109881 & 1.22216 & 0.0060216 \\
\hline & & & 21600 & 20833 & $>{ }^{4} \mathrm{I}_{13 / 2}$ & 0.035509 & 0.96449 & 0.188438 & 3.68163 & 0.0175945 \\
\hline \multicolumn{11}{|c|}{ Table 6. Thermal decomposition data for $\left[\mathrm{Ce}\left(\mathrm{NO}_{3}\right)_{3} 4[\mathrm{MCEABAB}]\right.$. } \\
\hline \multicolumn{2}{|c|}{ Compound } & \multicolumn{2}{|c|}{ Stage of decomposition } & \multicolumn{5}{|c|}{ Reaction Proposed } & \multicolumn{2}{|c|}{ Peak Temp. ${ }^{0} \mathrm{C}$} \\
\hline \multirow{4}{*}{\multicolumn{2}{|c|}{$\begin{array}{c}\mathrm{Ce}\left(\mathrm{NO}_{3}\right)_{3} \\
4[\mathrm{MCEABAB})\end{array}$}} & \multicolumn{2}{|c|}{ I } & \multicolumn{6}{|c|}{$\mathrm{Ce}\left(\mathrm{NO}_{3}\right)_{3} 4(\mathrm{MCEABAB}) \rightarrow \mathrm{Ce}\left(\mathrm{NO}_{3}\right)_{3} 3.07(\mathrm{MCEABAB})$} & 204 \\
\hline & & II & & \multicolumn{6}{|c|}{$\mathrm{Ce}\left(\mathrm{NO}_{3}\right)_{3} 3.07(\mathrm{MCEABAB}) \rightarrow \mathrm{Ce}\left(\mathrm{NO}_{3}\right)_{3} 1.23(\mathrm{MCEABAB})$} & 259 \\
\hline & & III & & \multicolumn{6}{|c|}{$\mathrm{Ce}\left(\mathrm{NO}_{3}\right)_{3} 1.23(\mathrm{MCEABAB}) \rightarrow \mathrm{Ce}\left(\mathrm{NO}_{3}\right)_{3} \quad 0.9(\mathrm{MCEABAB})$} & 407 \\
\hline & & IV & & \multicolumn{6}{|c|}{$\mathrm{Ce}\left(\mathrm{NO}_{3}\right)_{3} 0.9(\mathrm{MCEABAB}) \rightarrow \mathrm{Ce}_{2} \mathrm{O}_{3}$} & 598 \\
\hline
\end{tabular}


Table 7. Thermal decomposition data for $\left[\mathrm{Gd}\left(\mathrm{NO}_{3}\right)_{3} 4[\mathrm{MCEABAB}]\right.$.

\begin{tabular}{|c|c|c|c|}
\hline Compound & Stage of decomposition & Reaction Proposed & Peak Temp. ${ }^{0} \mathrm{C}$ \\
\hline \multirow{5}{*}{$\mathrm{Gd}\left(\mathrm{NO}_{3}\right)_{3} 4[\mathrm{MCEABAB})$} & I & Gd $\left(\mathrm{NO}_{3}\right)_{3} 4(\mathrm{MCEABAB}) \rightarrow \mathrm{Gd}\left(\mathrm{NO}_{3}\right)_{3} 3.77(\mathrm{MCEABAB})$ & 36 \\
\hline & II & $\mathrm{Gd}\left(\mathrm{NO}_{3}\right)_{3} 3.77(\mathrm{MCEABAB}) \rightarrow \mathrm{Gd}\left(\mathrm{NO}_{3}\right)_{3} 2.39(\mathrm{MCEABAB})$ & 259 \\
\hline & III & $\mathrm{Gd}\left(\mathrm{NO}_{3}\right)_{3} 2.39(\mathrm{MCEABAB}) \rightarrow \mathrm{Gd}\left(\mathrm{NO}_{3}\right)_{3} 1.68(\mathrm{MCEABAB})$ & 295 \\
\hline & IV & $\mathrm{Gd}\left(\mathrm{NO}_{3}\right)_{3} 1.68(\mathrm{MCEABAB}) \rightarrow \mathrm{Gd}\left(\mathrm{NO}_{3}\right)_{3} \quad 1.0(\mathrm{MCEABAB})$ & 481 \\
\hline & V & $\mathrm{Gd}\left(\mathrm{NO}_{3}\right)_{3} 1.0(\mathrm{MCEABAB}) \rightarrow \mathrm{Gd}_{2} \mathrm{O}_{3}$ & 775 \\
\hline
\end{tabular}

Table 8. Antifungal activities of some of the representative complexes of lanthanide(III) metal ions with Schiff base ligands (After 48 hours of incubation).

\begin{tabular}{|c|c|c|c|c|c|c|c|c|c|c|c|c|c|}
\hline \multirow{2}{*}{ S.No } & \multirow{2}{*}{ Complexes } & \multicolumn{4}{|c|}{ Species: Aspergillus fumigatus } & \multicolumn{4}{|c|}{ Species: Aspergillus niger. } & \multicolumn{4}{|c|}{ species: Mucor } \\
\hline & & 0 & $\begin{array}{c}1 \mathrm{mg} / 10 \\
\mathrm{~mL}\end{array}$ & $\begin{array}{c}2 \mathrm{mg} / 10 \\
\mathrm{~mL}\end{array}$ & $\begin{array}{c}3 \mathrm{mg} / 10 \\
\mathrm{~mL}\end{array}$ & 0 & $\begin{array}{c}1 \mathrm{mg} / 10 \\
\mathrm{~mL}\end{array}$ & $\begin{array}{c}2 \mathrm{mg} / 10 \\
\mathrm{~mL}\end{array}$ & $\begin{array}{c}3 \mathrm{mg} / 10 \\
\mathrm{~mL}\end{array}$ & 0 & $\begin{array}{c}1 \mathrm{mg} / 10 \\
\mathrm{~mL}\end{array}$ & $\begin{array}{c}2 \mathrm{mg} / 10 \\
\mathrm{~mL}\end{array}$ & $\begin{array}{c}3 \mathrm{mg} / 10 \\
\mathrm{~mL}\end{array}$ \\
\hline 1 & {$\left[\mathrm{Ce}(\mathrm{MCEABFAB})_{4}\left(\mathrm{NO}_{3}\right)_{3}\right]$} & $0.5 \mathrm{~cm}$ & $0.5 \mathrm{~cm}$ & $0.3 \mathrm{~cm}$ & $0.2 \mathrm{~cm}$ & - & - & - & - & $2.0 \mathrm{~cm}$ & $2.0 \mathrm{~cm}$ & $1.8 \mathrm{~cm}$ & $1.7 \mathrm{~cm}$ \\
\hline 2 & {$\left[\mathrm{Sm}(\mathrm{MCEABFAB})_{4}\left(\mathrm{NO}_{3}\right)_{3}\right]$} & $0.5 \mathrm{~cm}$ & $0.2 \mathrm{~cm}$ & $0.0 \mathrm{~cm}$ & $0.0 \mathrm{~cm}$ & $0.5 \mathrm{~cm}$ & $0.0 \mathrm{~cm}$ & $0.0 \mathrm{~cm}$ & $0.0 \mathrm{~cm}$ & $0.15 \mathrm{~cm}$ & $0.5 \mathrm{~cm}$ & $0.0 \mathrm{~cm}$ & $0.0 \mathrm{~cm}$ \\
\hline 3 & {$\left[\mathrm{Gd}(\mathrm{MCEABAB})_{4}\left(\mathrm{NO}_{3}\right)_{3}\right]$} & $1.0 \mathrm{~cm}$ & $1.0 \mathrm{~cm}$ & $0.3 \mathrm{~cm}$ & $0.2 \mathrm{~cm}$ & $1.0 \mathrm{~cm}$ & $0.5 \mathrm{~cm}$ & $0.2 \mathrm{~cm}$ & $0.2 \mathrm{~cm}$ & $2.0 \mathrm{~cm}$ & $1.0 \mathrm{~cm}$ & $0.5 \mathrm{~cm}$ & $0.5 \mathrm{~cm}$ \\
\hline
\end{tabular}

Table 9. Antibacterial activities of some of the representative complexes of lanthanide(III) metal ions with Schiff base ligands (After 48 hours of incubation).

\begin{tabular}{cccccc}
\hline \multirow{2}{*}{ S.No. } & Complexes & \multicolumn{4}{c}{ Species: E. coli } \\
\cline { 3 - 6 } & & 0 & $1 \mathrm{mg} / 10 \mathrm{~mL}$ & $2 \mathrm{mg} / 10 \mathrm{~mL}$ & $3 \mathrm{mg} / 10 \mathrm{~mL}$ \\
\hline 1 & {$\left[\mathrm{La}(\mathrm{MCEABAB})_{4}\left(\mathrm{NO}_{3}\right)_{3}\right]$} & $0.0 \mathrm{~cm}$ & $0.0 \mathrm{~cm}$ & $0.0 \mathrm{~cm}$ & $0.0 \mathrm{~cm}$ \\
2 & {$\left[\mathrm{La}(\mathrm{MCEABFAB})_{4}\left(\mathrm{NO}_{3}\right)_{3}\right]$} & $0.0 \mathrm{~cm}$ & $1.0 \mathrm{~cm}$ & $0.5 \mathrm{~cm}$ & $0.0 \mathrm{~cm}$ \\
3 & {$\left[\mathrm{Ce}(\mathrm{MCEABAB})_{4}\left(\mathrm{NO}_{3}\right)_{3}\right]$} & $0.0 \mathrm{~cm}$ & $0.0 \mathrm{~cm}$ & $0.0 \mathrm{~cm}$ & $0.1 \mathrm{~cm}$ \\
4 & {$\left[\mathrm{Sm}(\mathrm{MCEABAB})_{4}\left(\mathrm{NO}_{3}\right)_{3}\right]$} & $0.2 \mathrm{~cm}$ & $0.2 \mathrm{~cm}$ & $0.0 \mathrm{~cm}$ & $0.1 \mathrm{~cm}$ \\
\hline
\end{tabular}




\section{Configuration of the complexes}

The preferred coordination number for lanthanide(III) metal ions is either 6,8 or 10 . These Coordination numbers depend upon the nature of the anions present. Conductance and molecular weight data show that all the nitrate ions are present inside the coordination sphere in the complexes. IR spectral data also reveal that all nitrate ions are bi-dentately covalently bonded to lanthanide metal ions in all the complexes studied. Hence coordination number 10 is suggested for metal ion in these lanthanide(III) nitrate complexes ${ }^{34-35}$. The possible structure of the complexes is given Figure 2.

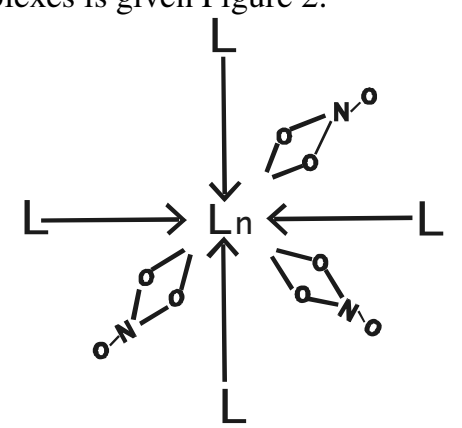

$[\mathrm{Ln}=\mathrm{La}, \mathrm{Ce}, \mathrm{Sm} \& \mathrm{Gd}, \mathrm{L}=\mathrm{MCEABAB}, \mathrm{MCEABCAB}, \mathrm{MCEABPT}$ and MCEABFAB $]$ (C.N. = 10)

\section{References}

Figure 2. The structure of the complexes

1. Huneey J E, Keiter E A and Keiter R L, Inorganic Chemistry Principles of Structure and Reactivity, Pearson Education, Singapore, 2002.

2. Kleber E V, Rare Earth Research, Macmillan, New York, 1961.

3. Moeller T, The Chemistry of Lanthanides, Reinhold, New York, 1963.

4. Cotton F A and Wilkinson G, Advanced inorganic Chemistry, $3^{\text {rd }}$ Edn., Interscience New York., 1972.

5. Koppikar D K, Sivapulliah P V, Ramakrishna L and Soundararajan S, Structure and Bonding, 1978, 34, 135.

6. Bradley J G, Gothra J S and Hart F A, J Chem Soc Dalton, 1973, 1021.

7. Harrison E, Acta Chem Scand., 1975, 27, 2827.

8. Kuma H and Yamada S, Inorg Chim Acta, 1975, 16, 213.

9. Mittal S P, Singh RV and Jadon J P, Curr Sc., (India), 1980, 49, 130.

10. Agarwal S K and Tandon J P, J Inorg Nucl Chem., 1975, 37, 1994.

11. Agarwal S K and Tandon J P, Montash Chem., 1979, 110, 401.

12. Arora K and Sharma K P, Synth.React.Inorg Met Org Chem., 2002, 32(5), 913.

13. Sharma K P, Jolly V S and Pathak P, Ultra Science, 1998, 10(2), 263.

14. Kishor Arora and Kiran Burman, J Saudi Chem Soc., 2007, 11(3), 445.

15. Chakravartrhi I, Ray H C, Goel N and Hashmi N M, Asian J Chem., 2002, 14, 143.

16. Agarwal R K and Himanshu Agarwal, Synth React Inorg Met Org Chem., 2001, 31, 263.

17. Indersenan P and Raj N K Kala, J Indian Chem Soc., 2000, 77, 259.

18. AgarwalR K, Arora K, Priyanka and Chakravarthi I, Polish J Chem., 1993, 67, 1913.

19. Radhakrishnan P S and Indersenan P, J Indian Chem Soc., 1990, 67, 243.

20. Shankar G, Prem Kumar R R and Ramalingham S K, Polyhedron, 1986, 5, 991.

21. Burns G R, Inorg Chem., 1968, 7, 277. 
22. Swaminathan K and Irving N H, J Inorg Nucl Chem., 1964, 261291.

23. Randle R R and Wiffen D H, Molecular Spectroscopy, Report of the conference of institute of Petroleum, Oct 1954.

24. Bullock J I, J Inorg Nucl Chem., 1967, 29, 2257.

25. Addison C C and Logan N, Advanced Inorg Chem Radiochem., 1964, 6, 95.

26. Lever A B P, Mantiovani E and Ramaswamy B S, Can J Chem., 1971, 49, 1957.

27. Dutt N K and Rahut S, J Inorg Nucl Chem., 1970, 32, 2105.

28. Butter E, Chem Abstr., 1972, 77, 8309300.

29. Kh A Cherches, Ezerskaya T P and Yalazere M, Chem Abstr., 1971, 74, 105621.

30. Lapitskaya A V and Pirkers S B, Zh Neorg Khim., 1971, 16, 369.

31. Tondon S P and Mehta P C, J Chem Phys., 1970, 52, 4314.

32. Kishor Arora and Brijesh Kumar Sharma, Asian J Chem., 1998, 10(4), 1032.

33. Agarwal R K and Arora K, Polish J Chem., 1993, 67, 25.

34. Agarwal R K, Kishor Arora and Sarin R K, Synth React Inorg Met Org Chem., 1994, 24(5) 735 .

35. Agarwal R K and Gupta S K, J Indian Chem Soc., 1986, 63, 994. 


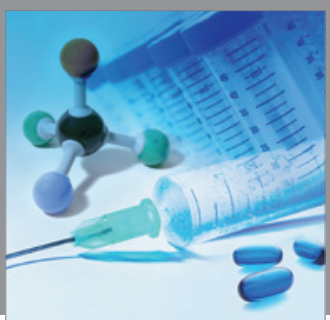

International Journal of

Medicinal Chemistry

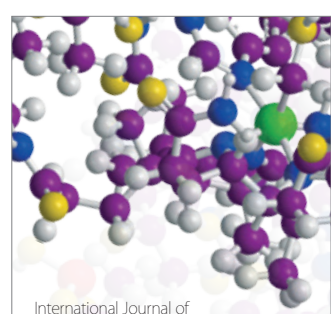

Carbohydrate Chemistry

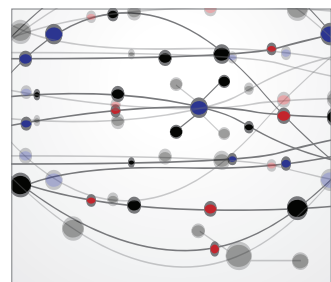

The Scientific World Journal
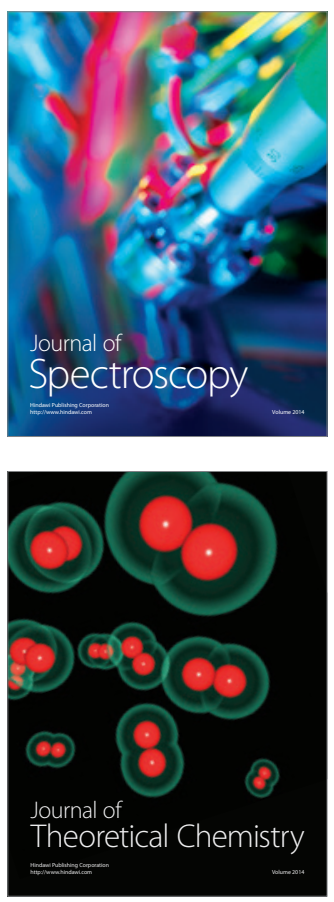
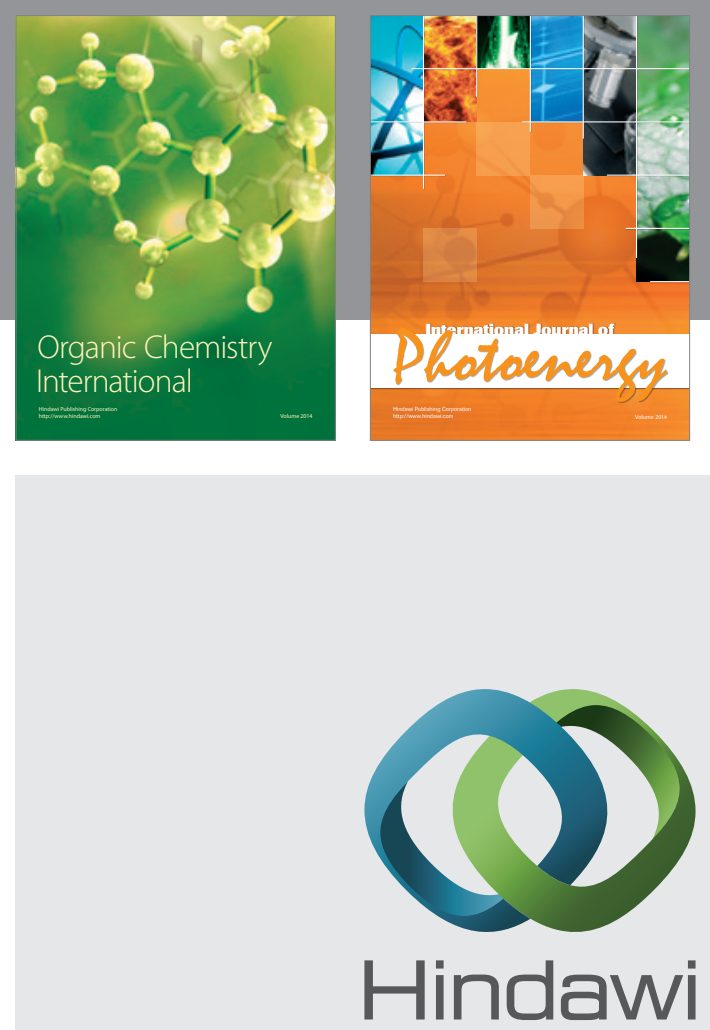

Submit your manuscripts at

http://www.hindawi.com
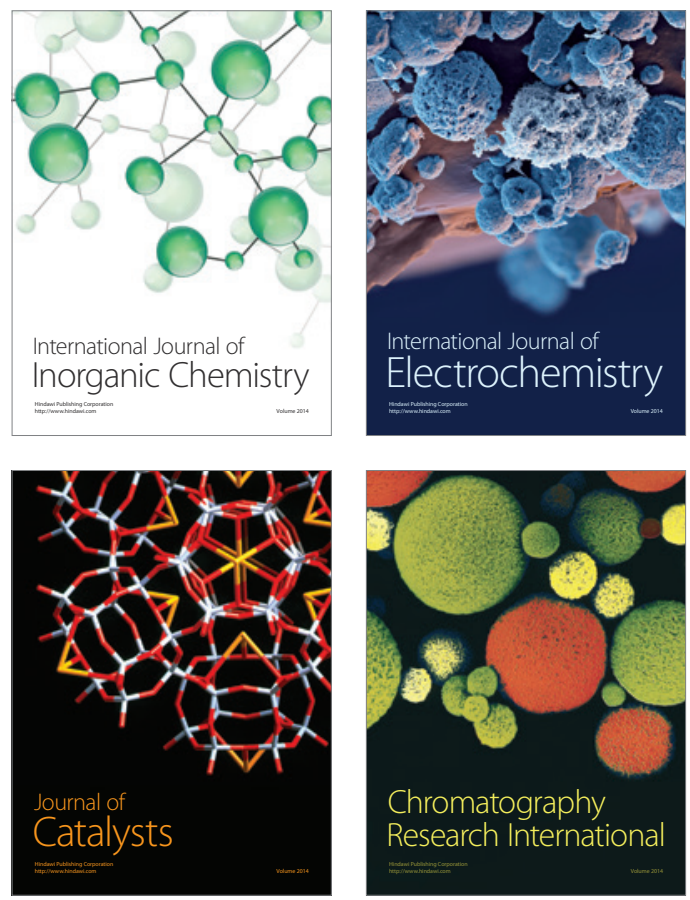
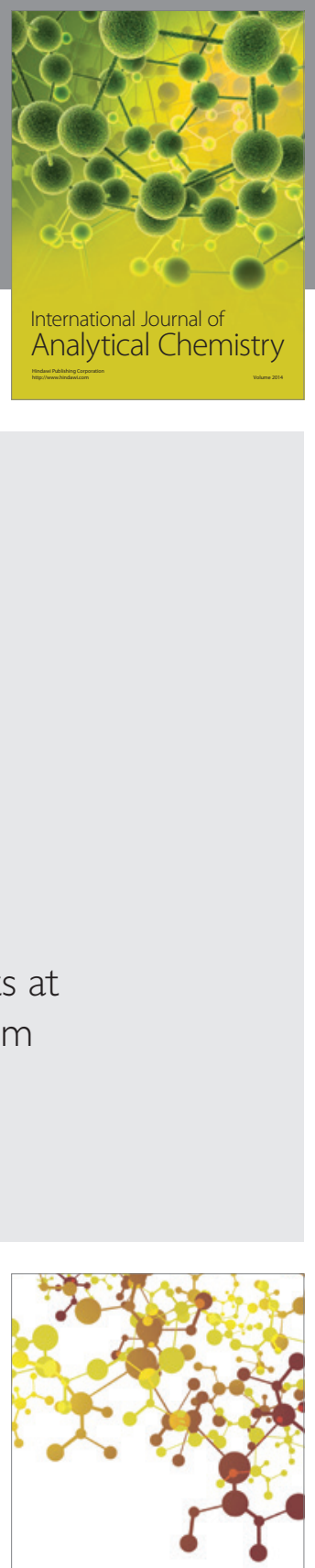

Journal of

Applied Chemistry
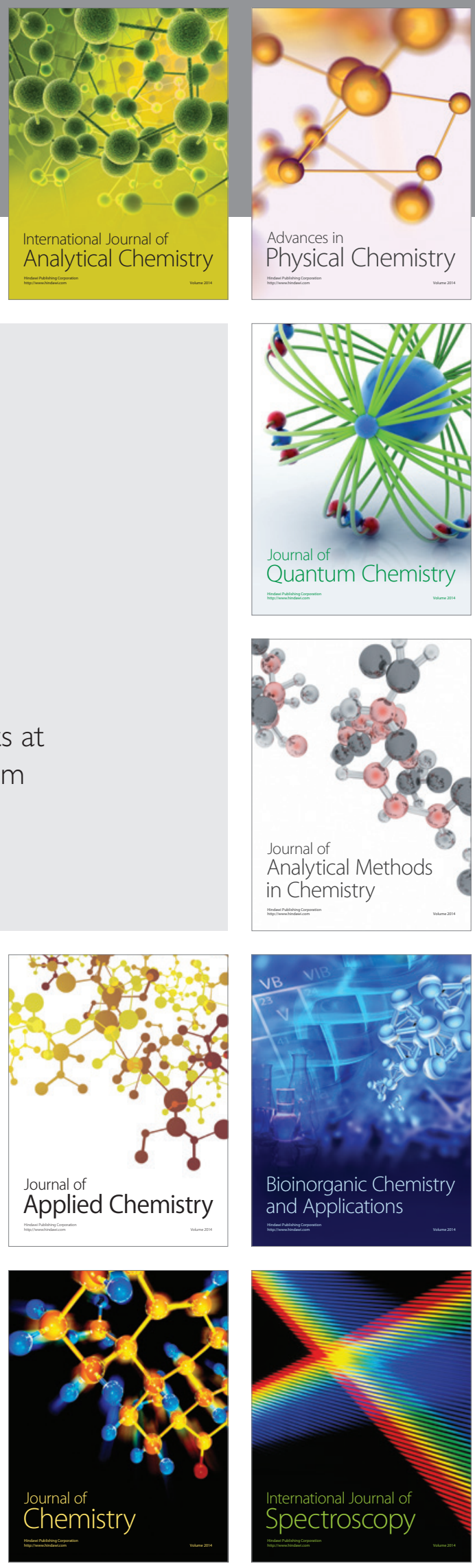